\title{
Corrigendum
}

\section{Research progress on space charge characteristics in polymeric insulation}

\author{
[Journal of Advanced Dielectrics, Vol. 6, No. 1 (2016) DOI: 10.1142/S2010135X16300012] \\ Yibo Zhang**, Thomas Christen ${ }^{\dagger}$, Xing Meng*, Jiansheng Chen* and Jens Rocks ${ }^{\dagger}$ \\ *ABB (China) Limited, Corporate Research, UBP B6L1 10 Jiuxianqiao Lu \\ Chaoyang District, Beijing 100015, P. R. China \\ ${ }^{\dagger} A B B$ Schweiz AG, Corporate Research, Segelhofstrasse $1 \mathrm{~K}, \mathrm{CH}-5405$ \\ Baden 5 Dättwil, Switzerland \\ tboveri-yibo.zhang@cn.abb.com \\ Published 27 December 2016
}

\begin{abstract}
We apologize for the following misprints in the review paper with title "Research progress on space charge characteristics in polymeric insulation", published in Vol 6./No. 1 of the "Journal of Advanced Dielectrics".

(1) In the citation of Fig. 6, Ref. 5 should be replaced by Ref. 32.

(2) In Fig. 10, Ref. 119 should be replaced by Ref. 120.

(3) In Table 1, Ref. 20 should be replaced by data partially from Ref. 35.

(4) On Page 5, left column, first paragraph of Part 3.3, last sentence "See Fig. 6 as an example for change density ..." "change" should be "charge".

(5) On Page 9, right column, last paragraph, "case-by case" should be "case-by-case".
\end{abstract}

